Results: One hundred and nine patients (mean age $54.7 \pm 18.5$ years, $78 \%$ women and $50.5 \%$ residents in rural areas) made 113 claims. The main reasons for claiming were: "advance medical evaluation and/or tests" (45.1\%), "request for evaluation by a specific physician" $(24.8 \%)$ and "rejection of evaluation by specific physician" (13, 3\%). $67 \%$ of the claims were satisfied. The least satisfied claims were "request for evaluation by specific doctor" (39.3\%) while claims for "cancellation of evaluation" were satisfied in $88 \%$ of the cases.

Median time between claim and previous medical visit was 99 [34-203] days and between claim and the subsequent medical consultation was 44 [28-82] days. Patients whose claims were due to "unpleasant attendance" were the first to complaint (median 28 [2-72] days) but waited for the longest time to be attended (median 103 [46-147] days). "Cancellation of evaluation" was attended after a median of 31.5 [28-33] days.

The main reasons for medical evaluation were: inflammatory/systemic disease in 25 patients $(22.15 \%)$, neck and low back pain in 23 patients $(20.35 \%$ ) and FM/CWP also in $20.35 \%$ of patients. As personal medical history, Psychiatric Disorders were present in $20.5 \%$ of patients and multiple comorbidities in $19.6 \%$ of patients. Twenty patients had no medical history of interest.

Regarding the type of claims, differences were observed related to the diagnosis and the patient's medical history. The logistic regression model (FM as dependent variable) adjusted for sex, age, rural area, time to/ after the claim and medical history showed that patients with FM/CWP requested more frequently to "be evaluated by other physician" (OR of 23.92 ( $95 \% \mathrm{Cl}, 1.4-409.06)$ and "reject to be evaluated by a specific physician" (OR of $8.48(95 \% \mathrm{Cl}, 1.2-60.09)$ than rest of the patients and also presented more frequently with psychiatric history (OR of 22.39 $(95 \% \mathrm{Cl}, 1.15-437.23)$

Conclusion: the present study reflects main reasons for claiming of patients treated in a rheumatology department. Reasons of claiming and comorbidities of patients with FM/CWP differ from those of the rest of patients. These findings may be of interest for the organization of resources in rheumatology departments.

Disclosure of Interests: Enrique Judez Navarro Consultant for: Roche, Carlos Sánchez-Piedra: None declared, Gines Sanchez Nievas: None declared, Isabel Labiano: None declared, Manuela Sianes: None declared, Gloria Garcia Consuegra: None declared, Sandra Soro: None declared, M Angeles Garcia Morales: None declared

DOI: 10.1136/annrheumdis-2019-eular.8243

\section{AB1218 ANALYSIS OF REFERRAL TRENDS TO A SECONDARY CARE RHEUMATOLOGY SERVICE FROM GENERAL PRACTICE,OVER A TEN- YEAR PERIOD}

Ganesh Kasavkar, Kaushik Chaudhuri, Roxanne Mcvittie. University Hospital Coventry and Warwickshire, Rheumatology, Coventry, United Kingdom

Background: The practice of Rheumatology in the UK has undergone significant change in the past decade. These have been driven by developments in our understanding of the diseases; early arthritis clinics; changing population demographics; changes in the health services (NHS) all of which have influenced referral patterns to rheumatology.

Objectives: To analyse referral data from primary care three time points of a month at a time, over a decade - from 2006 to 2016 to assess changing trends, if any, of patients attending Rheumatology.

Methods: We analysed referrals from the primary care in the months of Feb '06 (n=89), Aug'12 $(n=50)$ and Feb'16 $(n=100)$. We looked at the following: reason for referral and our response, referral to out-patient review interval; outcome and final diagnosis.

We also analysed between first appointment and DMARD initiation in inflammatory arthritis.

Results: Referrals were grouped as demonstrated in the table below.

\begin{tabular}{lccc}
\hline Diagnostic category & $2006(n=89)$ & $2010(n=50)$ & $2016(n=100)$ \\
\hline non inflammatory/inflammatory arthritis & $82 \%(n=73)$ & $68 \%(n=34)$ & $73 \%(n=73)$ \\
Abnormal investigations results for opinion & $2.5 \%(n=2)$ & $6 \%(n=3)$ & $10 \%(n=10)$ \\
Back pain & $11.3 \%(n=10)$ & $10 \%(n=5)$ & $5 \%(n=5)$ \\
Myalgia & $1.1 \%(n=1)$ & $10 \%(n=5)$ & $3 \%(n=3)$ \\
Suspected connective tissue disease & $1.1 \%(n=1)$ & $4 \%(n=2)$ & $6 \%(n=6)$ \\
Soft tissue conditions/others & $2.2 \%(n=2)$ & $2 \%(n=1)$ & $3 \%(n=3)$ \\
\hline
\end{tabular}

The mean interval from Referral to patient review was 11.5 weeks in Feb 2006, 7.15 weeks in August 2012 and 6.6 weeks in Feb 2016. In patients with the diagnosis of inflammatory arthritis we also looked at mean timeframe from $1^{\text {st }}$ review to initiation of DMARDs which is demonstrated in the chart below.

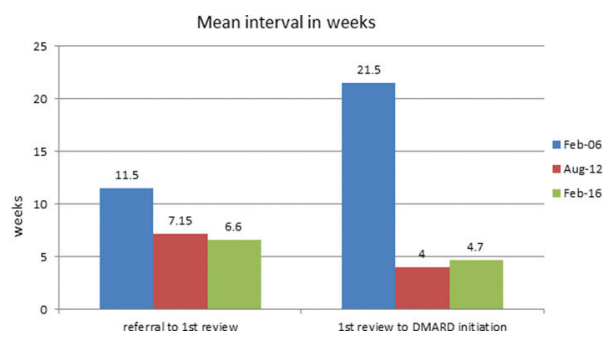

Figure 1

Conclusion: - Mean interval between primary care referral to first outpatient review has improved significantly over the period of 10 years

- Mean interval for DMARD initiation in inflammatory arthritis patients has also improved in the 10 years' time

Disclosure of Interests: None declared

DOI: 10.1136/annrheumdis-2019-eular.8282

\section{AB1219 OF INDIVIDUALS WITH MUSCULOSKELETAL COMPLAINTS: A PROSPECTIVE COHORT STUDY}

Harald Leiss ${ }^{1}$, Miriam Hucke ${ }^{2}$, Veronika Machold-Fabrizii ${ }^{3}$, Josef S. Smolen ${ }^{1}$, Klaus Machold ${ }^{1}$. ${ }^{1}$ Medical University of Vienna, Department of Rheumatology, Vienna, Austria; ${ }^{2}$ Klinikum Klagenfurt, Department of Internal Medicine and Gastroenterology, Hepatology, Endocrinology and Nephrology, Klagenfurt, Austria; ${ }^{3}$ Wilhelminenspital, 6th Medical Department with Nephrology and Dialysis, Vienna, Austria

Background: Employed people with musculoskeletal complaints often seek medical advice when symptoms are chronic and lead to loss of workability.

Objectives: A brief examination was offered in the workplace setting to detect and to counsel individuals with symptoms of Rheumatic and musculoskeletal diseases(RMDs).

Methods: Employees received a questionnaire regarding musculoskeleta problems. In case of a positive screening, consultation by RMD specialists was offered. If necessary, participants were referred to a clinic specialized in RMDs. Employees' work was categorized into physically-highly demanding(HD) and less-demanding(LD).

Following data were acquired: demographics, known pre-existing RMD pain intensity, affected region(s), current treatment, number of sick-leavedays due to musculoskeletal complaints, and out of pocket costs for treatments during the preceding year. General wellbeing and depression were measured by Euroquol-5d. Follow-up information about data mentioned above was collected by telephone-interview.

Results: 6170 employees were invited. 413 participated in the counselling program, 344 were enrolled in the study. $56.6 \%$ of the participants had no previously diagnosed RMD, after the specialists' assessment, this percentage decreased to $35.7 \%$. Men with LD-workload had significantly higher wellbeing (EQ-5d scale) compared to women with both LD $(p=0.034)$ and $H D(p=0.001)$. LD and HD differed significantly regarding percentage with painful upper $(p=0.006)$ and lower $(p=0.016)$ limbs. Back pain was distributed equally among all groups. HD women reported significantly higher use of NSAIDs $(p=0.001)$.

235 individuals participated in telephone follow-up. There was significant improvement in wellbeing $(p=0.006)$ and in rating of $\operatorname{RMD}$ pain $(p=0.001)$ Participants who were suspected to suffer from RMDs had significantly increased out of pocket costs after one year $(p=0.026)$. Use of NSAIDs decreased significantly from 29.1 to $17.4 \% \quad(p=0.02)$. Rates of use of physiotherapy $(p=0.001)$, gymnastics $(p=0.001)$, physical therapy $(p=0.027)$ and complementary/alternative methods $(p=0.003)$ were significantly increased.

Conclusion: We found most physical/psychological problems related to RMDs in HD-working women. After one year, participants reported improved quality of life, reduction of RMD pain, higher utilization of medical services and of gymnastics, less use of NSAIDs, and, if suspected to suffer from RMDs, higher out of pocket costs. Thus, this workplacecentered intervention appears to have beneficial effects on both subjective well-being and physical/physiological health.

Disclosure of Interests:: Harald Leiss Consultant for: Lilly, MSD, Miriam Hucke Grant/research support from: Abbvie, Veronika Machold-Fabrizii: None declared, Josef S. Smolen Grant/research support from: AbbVie, El Lilly, Janssen, MSD, Pfizer, Roche, Consultant for: AbbVie, Amgen, Astra- 